\title{
Autoimmune lymphoproliferative syndrome due to CTLA4 haploinsuffiency
}

INSERM

\section{Source}

INSERM. (1999). Orphanet: an online rare disease and orphan drug data base.

Autoimmune lymphoproliferative syndrome due to CTLA4 haploinsuffiency.

ORPHA:436159

Autoimmune lymphoproliferative syndrome due to CT LA4 haploinsuffiency is a rare, primary immunodeficiency characterized by variable combination of enteropathy, hypogammaglobulinemia, recurrent respiratory infections, granulomatous lymphocytic interstitial lung disease, lymphocytic infiltration of non-lymphoid organs (intestine, lung, brain, bone marrow, kidney), autoimmune thrombocytopenia or neutropenia, autoimmune hemolytic anemia and lymphadenopathy. 